$$
K M \equiv 0\left(\bmod 2^{n-1} \cdot 9\right) .
$$

Conversely (11) implies (9). Since (9) holds for the modulus $2^{n-2} \cdot 9 M$, it follows similarly that (11) holds for the modulus $2^{n-2} .9$ with $M=2^{n-4} M_{1}$. Hence (11) will be true for the given modulus if $M=2^{n-3} M_{1}$. This supplies a proof by induction that (8) is a universal form for every $n \geqq 4$.

If, in addition, ${ }^{*} M$ is divisible by every prime $p$ where $3<p \leqq n$, we satisfy the necessary condition given by Dickson $\dagger$ for the form (8) to represent at least one set of $n$ primes. The proof of the sufficiency of this condition still remains a challenge to the ingenuity of number theorists.

NEW YORK, N. Y.

\title{
RINGS AS GROUPS WITH OPERATORS
}

\section{J. EVERETT, JR.}

1. Introduction. A module $M(0, a, b, \cdots)$ is a commutative group, additively written. Every correspondence of $M$ onto itself, or part of itself, such that $a \rightarrow a^{\prime}, b \rightarrow b^{\prime}$ implies $a+b \rightarrow a^{\prime}+b^{\prime}$ defines an endomorphism of $M$. An endomorphism may be regarded as an operator $\theta$ on $M$ subject to the postulates (i) $\theta a=a^{\prime}$ is uniquely defined as an element of $M$, (ii) $\theta(a+b)=\theta a+\theta b,(a, b \varepsilon M)$. In particular, there exist a null operator $0(0 M=0)$ and a unit operator $\epsilon(\epsilon a=a, a \varepsilon M)$. Designate by $\Omega_{M}$ the set of all such operators, $0, \epsilon, \alpha, \beta, \ldots$. It is well known that if operations of $\oplus$ and $\odot$ be defined in $\Omega_{M}$ by $(\theta+\eta) a=\theta a+\eta a$ and $(\theta \eta) a=\theta(\eta a),(a \varepsilon M), \Omega_{M}$ forms a ring with unit element $\epsilon$ (endomorphism ring of $M$ ). $\ddagger$ The equation $\theta=\eta$ means $\theta a=\eta a$ (all $a \varepsilon M)$. A ring $R(M)$ is called a ring over $M$ in case $M$ is the additive group of $R(M)$. Correspondence of a set $P$ onto a set $Q$ (many-one) is written $P \sim Q$; if specifically one-one, $P \cong Q$. Corresponding operations in $P, Q$ preserved under the map are indicated in parentheses; for example, $P \sim Q(+)$. If a set $T$ has the property that $T P$ is defined in $P, T Q$ in $Q$, and if, under a correspondence $P \sim Q, p \rightarrow q$ implies $t p \rightarrow t q(t \varepsilon T, p \varepsilon P, q \varepsilon Q)$, we write $P \sim Q(T)$ ( $T$-operator correspondence). If $R$ is a ring, the two-sided ideal $N$ of elements $z$ of $R$ such that $z r=0$ (all $r \varepsilon R$ ), is called the left annulling ideal of $R$.

* For example, replace $6 M$ in (8) by $2^{w} n ! M,(w \geqq n-3)$.

$\dagger$ Loc. cit., p. 156.

$\ddagger$ van der Waerden, Moderne Algebra, vol. 1, 2d edition, p. 146. 
2. Fundamental theorems. We prove first the following theorem:

TheOREM 1. If $R(M)$ is a ring over $M$, there exists in $\Omega_{M}$ a subring $\Gamma$ such that

$$
R(M) \sim \Gamma(\oplus, \odot ; \Gamma),
$$

this correspondence being one-one if and only if $N=(0)$ for $R(M) .^{*}$

For $R(M)$ consists of the elements of $M$ on which a multiplication has been defined so that (i) $a b \varepsilon M$, (ii) $a(b+c)=a b+a c$, (iii) $(a+b) c$ $=a c+b c$, (iv) $(a b) c=a(b c)$. By (i), every $a$ of $M$ defines a map of $M$ in to $M$ which by (ii) is an endomorphism. Hence to every $a$ of $M$ corresponds an operator $\alpha$ of $\Omega_{M}$. Let $\Gamma$ be the set of all such $\alpha$, whence $R(M) \sim \Gamma$, where $a \rightarrow \alpha$ is defined by $a g=\alpha g$ (all $g \varepsilon M)$. We have that $a+b \rightarrow \alpha+\beta, a b \rightarrow \alpha \beta$ and $\gamma a \rightarrow \gamma \alpha$ from the following:

$$
\begin{aligned}
(a+b) h & =a h+b h=\alpha h+\beta h=(\alpha+\beta) h, \\
(a b) h & =a(b h)=a(\beta h)=\alpha(\beta h)=(\alpha \beta) h, \\
(\gamma a) h & =(g a) h=g(a h)=(\gamma \alpha) h,
\end{aligned}
$$
all $h \varepsilon M$.

Since, under the correspondence, $N \rightarrow 0$, proof of the theorem is complete.

THEOREM 2. If in $\Omega_{M}$ there exists a subring $\Gamma$ such that $M \sim \Gamma(\oplus ; \Gamma)$ then there exists a ring $R(M)$ over $M$ such that

$$
R(M) \sim \Gamma \quad(\oplus, \odot ; \Gamma) .
$$

We define $a b=\alpha b$. Then

$$
\begin{aligned}
a(b+c) & =\alpha(b+c)=\alpha b+\alpha c=a b+a c, \\
(a+b) c & =(\alpha+\beta) c=\alpha c+\beta c=a c+b c, \\
(a b) c & =(\alpha b) c=(\alpha \beta) c=\alpha(\beta c)=\alpha(b c)=a(b c),
\end{aligned}
$$

and $M$ with this multiplication is a ring $R(M)$. Since $a b=\alpha b \rightarrow \alpha \beta$, the theorem follows.

Corollary. If $M \sim \Gamma(\oplus), \Gamma$ a submodule of $\Omega_{M}$, there exists a (nonassociative) ring $R^{*}(M)$ over $M$, where $a b$ is defined as $\alpha b,(a \rightarrow \alpha)$.

The relation between associativity of $R(M)$ and the $\Gamma$-operator character of the correspondence seems to indicate a point of departure for the study of rings with associativity not assumed.

* In case $N \neq(0)$, there exists a ring $R_{1} \supset R$ for which $N_{1}=(0)$; thus $R$ is always isomorphic with a subring of the endomorphism ring of some module. See, for example, A. A. Albert, Modern Higher Algebra, University of Chicago Press, 1937, p. 22, Theorem 5. 
3. On linear algebras. Let $V$ be a vector space of $n$ dimensions over a field $F$. Elements of $V$ satisfy

$$
\left(\begin{array}{c}
\alpha_{1} \\
\vdots \\
\alpha_{n}
\end{array}\right)=\left(\alpha_{i}\right)=\sum \alpha_{i} d_{i}, \quad\left(\alpha_{i}\right)+\left(\beta_{i}\right)=\left(\alpha_{i}+\beta_{i}\right), \quad \alpha\left(\alpha_{i}\right)=\left(\alpha \alpha_{i}\right) .
$$

It is well known* that every $F$-operator endomorphism of $V\left(v \rightarrow v^{\prime}\right.$ implies $\left.\alpha v \rightarrow \alpha v^{\prime}\right)$ is represented by an $n \times n$ matrix over $F$ operating on $V$. For under such a map, $d_{i} \rightarrow \sum \alpha_{j i} d_{j}$, and

$$
v=\sum \alpha_{i} d_{i} \rightarrow \sum\left(\sum \alpha_{i} \alpha_{j i}\right) d_{j}=A v,
$$

where $A$ is the matrix $\left(\alpha_{i j}\right)$. Now a linear associative algebra of order $n$ over the field $F$ is simply a ring $A(V)$ over $V$ subject to the axioms (i) $\alpha(u v)=u(\alpha v)$ and (ii) $\alpha(u v)=(\alpha u) v$. Condition (i) requires that the endomorphism defined by the multiplier $u$ be an $F$-operator map, that is, $u v=U v$, where $U$ is a matrix of the type just indicated. Hence in the correspondence of Theorem $1, u \rightarrow U$; and by (ii), $\alpha u \rightarrow \alpha U$, $(\alpha \varepsilon F)$. Thus

$$
A(V) \sim \Gamma \quad(\oplus, \odot ; \Gamma, F)
$$

where $\Gamma$ is a subalgebra of the total $n \times n$ matrix algebra $\mathcal{X}$ over $F$. This correspondence (which is the classical one) is biunique if and only if the left annulling ideal $N$ of $A(V)$ is (0), a much weaker condition than the possession of unit element usually required. The $\Gamma$ operator property of the correspondence is significant in the light of the following remark, which is in part a result of Theorem 2:

If $V \sim \Gamma(\oplus ; \Gamma, F), \Gamma$ any subalgebra of $\mathcal{X}$, then there exists an algebra $A(V)$ over $V$ such that

$$
A(V) \sim \Gamma \quad(\oplus, \odot ; \Gamma, F) .
$$

That not every matrix representation of an algebra possesses the $\Gamma$-operator property is evinced by the example

$$
A(V):\left(\begin{array}{c}
\alpha_{1} \\
\alpha_{2}
\end{array}\right),\left(\begin{array}{l}
\alpha_{1} \\
\alpha_{2}
\end{array}\right)\left(\begin{array}{l}
\beta_{1} \\
\beta_{2}
\end{array}\right)=\left(\begin{array}{ll}
\alpha_{1} & \beta_{1} \\
\alpha_{1} & \beta_{2}
\end{array}\right),
$$

for

but the relation

$$
\left(\begin{array}{l}
\beta_{1} \\
\beta_{2}
\end{array}\right) \cong\left(\begin{array}{cc}
\beta_{1} & \beta_{2} \\
0 & 0
\end{array}\right)(\oplus, \odot)
$$

* See van der Waerden, loc. cit., vol. 2, p. 111. 


$$
\left(\begin{array}{cc}
\alpha_{1} & \alpha_{2} \\
0 & 0
\end{array}\right)\left(\begin{array}{l}
\beta_{1} \\
\beta_{2}
\end{array}\right) \rightarrow\left(\begin{array}{cc}
\alpha_{1} & \alpha_{2} \\
0 & 0
\end{array}\right)\left(\begin{array}{cc}
\beta_{1} & \beta_{2} \\
0 & 0
\end{array}\right)
$$

does not hold. However

$$
\left(\begin{array}{l}
\beta_{1} \\
\beta_{2}
\end{array}\right) \sim \Gamma \equiv\left(\begin{array}{ll}
\beta_{1} & 0 \\
0 & \beta_{1}
\end{array}\right)(\oplus, \odot ; \Gamma) .
$$

4. Reduction theorems for finite rings. Let $M$ be a module of order $m=p_{1}{ }^{a_{1}} \cdots p_{n}{ }^{a_{n}}$. Then $M=B_{1}+\cdots+B_{n}$ is a direct sum, $B_{i}$ of order $p_{i}{ }^{{ }^{i}}$, containing all elements of period dividing $p_{i}{ }^{{ }^{i}}$. Moreover, $B_{i}=C_{i 1}+\cdots+C_{i l_{i}}$, where $C_{i j}$ is cyclic of order $p_{i}{ }^{b i j}, \sum_{j=1}^{l_{i}} b_{i j}=a_{i}$. The endomorphism ring $\Omega_{M}$ of $M$ is a direct sum of endomorphism rings of the $B_{i}$ :

$$
\Omega_{M}=\Omega_{1}+\cdots+\Omega_{n},
$$

$\Omega_{i}$ a two-sided ideal in $\Omega_{M}, \Omega_{i} \cap \Omega_{j}=\delta_{i j} \Omega_{j}, \Omega_{i} \Omega_{j}=\delta_{i j} \Omega_{i}{ }^{2}$. Further, if $B=C_{1}+\cdots+C_{l}, C_{j}$ of order $p^{b j}$, be represented as a vector space

$$
\left(\begin{array}{c}
x_{1} \\
\cdot \\
x_{l}
\end{array}\right), x_{j}\left(\bmod p^{b_{j}}\right), b_{1} \leqq \cdots \leqq b_{l},
$$

then $\Omega_{B}$ may be represented* by the ring of all matrices $\left(\beta_{j k}\right)$ $=\left(\alpha_{j k} p^{b_{j}-b_{k}}\right), p^{b_{j}-b_{k}}$ defined as 1 for $j<k, \beta_{j k}$ reduced $\left(\bmod p^{b_{j}}\right)$. Thus if $M$ is represented as a vector space, $\Omega_{M}$ is a ring of matrices with blocks along the diagonal, the $\Omega_{i}$-blocks having the $\left(\beta_{j k}\right)$ structure described. $\dagger$

Theorem 3. If $M \sim \Gamma \subset \Omega_{M}(\oplus ; \Gamma)$, then $\Gamma=\Gamma_{1}+\cdots+\Gamma_{n}$, a direct sum of two-sided ideals in $\Gamma$, and

$$
B_{i} \sim \Gamma_{i} \subset \Omega_{i}\left(\oplus ; \Gamma_{i}\right) .
$$

Let $\Gamma_{i}$ be the map of $B_{i}$. Then $\Gamma_{i}$ is a two-sided ideal in $\Gamma$, and every $\gamma \varepsilon \Gamma$ is a sum of $\gamma_{i} \varepsilon \Gamma_{i}$. Moreover $\Gamma_{i} \subset \Omega_{i}$. For let $b_{i} \rightarrow \lambda_{i} \varepsilon \Gamma_{i}$, $\left(\lambda_{i}=\left(\theta_{1}+\cdots+\theta_{n}\right), \theta_{i} \varepsilon \Omega_{i}\right)$. Since $b_{i} \varepsilon B_{i}$,

$$
p_{i}^{a_{i}} b_{i}=0 \rightarrow p_{i}^{a_{i}}\left(\theta_{1}+\cdots+\theta_{n}\right)=0 .
$$

Hence $p_{i}{ }^{a} \theta_{j}=0,(j=1, \cdots, n)$. From the structure of $\Omega_{i}$ already indicated, $\theta_{j}=0,(j \neq i)$. Thus $\Gamma$ is a direct sum.

* K. Shoda, Über die Automorphismen einer endlichen Abelschen Gruppe, Mathematische Annalen, vol. 100 (1928), p. 676.

$\dagger$ Note that $B$ is admissible relative to $\Omega_{M}$, that is, $\Omega_{M} B_{i} \subset B_{i}$. 
THEOREM 4. If $M=B_{1}+\cdots+B_{n}, B_{i} \sim \Gamma_{i}\left(\oplus ; \Gamma_{i}\right), \Gamma_{i}$ a subring of $\Omega_{i}$, then $\Gamma=\Gamma_{1}+\cdots+\Gamma_{n}$ is direct, $\Gamma_{i}$ a two-sided ideal in $\Gamma$, and

$$
M \sim \Gamma \subset \Omega_{M}(\oplus ; \Gamma) .
$$

Since $\Gamma_{i} \subset \Omega_{i}, \Gamma$ is a direct sum, and $\Gamma_{i}$ is a two-sided ideal in $\Gamma$. Define $M \sim \Gamma$ by $m=b_{1}+\cdots+b_{n} \rightarrow \gamma_{1}+\cdots+\gamma_{n}$ (where $b_{i} \rightarrow \gamma_{i}$ ). Then addition is preserved. Let $\rho \varepsilon \Gamma, \rho=\mu_{1}+\cdots+\mu_{n},\left(\mu_{i} \varepsilon \Gamma_{i}\right)$. Then

$$
\begin{aligned}
\rho m & =\rho b_{1}+\cdots+\rho b_{n}=\mu_{1} b_{1}+\cdots+\mu_{n} b_{n} \rightarrow \mu_{1} \gamma_{1}+\cdots+\mu_{n} \gamma_{n} \\
& =\left(\mu_{1}+\cdots+\mu_{n}\right)\left(\gamma_{1}+\cdots+\gamma_{n}\right) .
\end{aligned}
$$

THEOREM 5. Every ring over $M=B_{1}+\cdots+B_{n}$ is a direct sum of rings over the $B_{i}$; hence to construct all rings over $M$ it is only necessary to construct all rings over the $B_{i}$.

5. On elementary modules. $M$ is said to be elementary in case there exists an isomorphism

$$
M \cong \Omega_{M}\left(\oplus ; \Omega_{M}\right) .
$$

THEOREM 6. $M$ is elementary if and only if there exists a ring with unit element, $R(M)$ over $M$, such that every endomorphism of $M$ is defined by a left multiplier of $R(M)$.

For if $M$ is elementary, there exists a ring $R(M)$ such that

$$
R(M) \cong \Omega_{M}\left(\oplus, \odot ; \Omega_{M}\right)
$$

where $a b$ is defined as $\alpha b,(a \leftarrow \rightarrow \alpha)$. Let $m \rightarrow \theta m$ be an endomorphism of $M$. In the above isomorphism let $t \hookleftarrow \rightarrow$. Then $t m=\theta m,(t \varepsilon R(M))$. Conversely, if $R(M)$ is of this type,

$$
R(M) \cong \Gamma \subset \Omega_{M}(\oplus, \odot ; \Gamma),
$$

and if one assumes $\theta \varepsilon \Omega_{M}$, there exists a $t \varepsilon R(M)$ such that $t a=\theta a$, ( $a \varepsilon M$ ). Hence $\theta \varepsilon \Gamma$ and $\Gamma=\Omega_{M}$; whence $M$ is elementary.

COROLlary. The modules of rational numbers, and of rational integers $C$ (the infinite cyclic group) are elementary.

For it is readily shown that the only solution of the functional equation $\Phi=(a+b)=\Phi(a)+\Phi(b)$ in the field of rationals and the ring of integers is of the type $\Phi(a)=r a$ where $r$ is a multiplier of the domain.

COROLlaRY. The only rings $R(C)$ over $C$ are given by the multiplication $a \cdot b$, defined as any fixed positive integral multiple of the ordinary product $a b$ in the ring of rational integers. 
To define a ring $R(C)$ we must obtain a homomorphism

$$
C \sim \Gamma(\oplus ; \Gamma)
$$

where $\Gamma$ is a subring of $\Omega_{C}$, setting $a \cdot b=\alpha b(a \rightarrow \alpha)$. But $\Omega_{C}$ is the ordinary ring of rational integers, its only subrings being principal ideals $\{m\}$. Hence we must have

$$
C \sim\{m\}(\oplus ;\{m\})
$$

where $1 \rightarrow m, a \rightarrow m a$.

THeOREm 7. If $M$ is elementary, the units of $\Omega_{M}$ are in the centrum of $\Omega_{M} *^{*}$

For the endomorphism $\sigma^{-1} \Omega_{M} \sigma$ of the additive group of $\Omega_{M}(\sigma$ a unit) must be defined by a ring multiplier $\rho: \sigma^{-1} \Omega_{M} \sigma=\rho \Omega_{M}$. Then in particular $\sigma^{-1} \epsilon \sigma=\rho \epsilon$ and $\rho=\epsilon$.

Corollary. A vector space $V$ of order greater than or equal to 2 is not elementary.

For there always exist nonsingular matrices not commutative with the total matrix algebra, and hence not in the centrum of $\Omega_{V}$.

TheOREM 8. A finite module $M$ is elementary if and only if it is cyclic.

For a cyclic $M, \Omega_{M}$ is represented by the $n \times n$ matrices $\left(\delta_{i j} \alpha_{j}\right), \alpha_{j}$ $\left(\bmod p_{i}{ }^{a}\right)$. Hence under

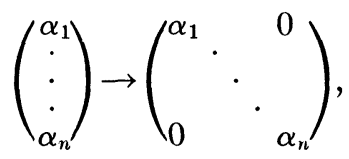

$M$ is elementary. If there are repeated primes in the type of $M$, then the order of $\Omega_{M}$ is greater than that of $M$ and $M$ is not elementary (see $\$ 4$ ).

Thus the rings $R(M)$ over elementary finite $M$ are completely known, $\left(\alpha_{i}\right)\left(\beta_{i}\right)$ being defined as $\left(\gamma_{i} \alpha_{i} \beta_{i}\right),\left(0 \leqq \gamma_{i}<p_{i}{ }^{a_{i}}\right)$.

UNIVERSITY OF WISCONSIN tive.

* A stronger theorem holds: If $M$ is elementary, its endomorphism ring is commuta- 